Submission to Review of Scientific Instruments (RSI)

\title{
AC modeling and impedance spectrum tests of the superconducting magnetic field coils for the Wendelstein 7-X fusion experiment.
}

\author{
Hartmut Ehmler* and Matthias Köppen \\ Max-Planck Institute for Plasma Physics, Wendelsteinstr. 1, D-17489 Greifswald, \\ Germany, EURATOM-Association \\ *corresponding author, formerly at Max-Planck Institute for Plasma Physics, Greifswald; \\ now at Hahn-Meitner Institut Berlin, Structural Research Division and Berlin Neutron \\ Scattering Center, High Field Magnet Project, Glienicker Str. 100, D-14109 Berlin, \\ phone: 0049-30-8062-3166, fax: 0049-30-8062-2548, e-mail: hartmut.ehmler@hmi.de
}

\begin{abstract}
The impedance spectrum test was employed for detection of short circuits within W7-X superconducting magnetic field coils. This test is based on measuring the complex impedance over several decades of frequency. The results are compared to predictions of appropriate electrical equivalent circuits of coils in different production states or during cold test. When the equivalent circuit is not too complicated the impedance can be represented by an analytic function. A more detailed analysis is performed with a network simulation code. The overall agreement of measured and calculated or simulated spectra is good. Two types of short circuits which appeared are presented and analyzed. The detection limit of the method is discussed. It is concluded that combined highvoltage $\mathrm{AC}$ and low voltage impedance spectrum tests are ideal means to rule out short circuits in the W7-X coils.
\end{abstract}




\section{Introduction}

Electric AC tests are generally carried out to control the quality of fabrication of magnetic coils. For the W7-X magnets [1],[2] three testing methods have been applied: High-voltage (HV) continuous AC test at fixed frequency, HV-impulse test, and lowvoltage impedance spectrum test [3]. In some magnetic field coils of W7-X short circuits in the winding were detected. Some of the coils were already in an advanced production state. The reason for this was that testing procedures were not sufficient at first but were developed during production and acceptance tests [4]. This paper focuses on the impedance spectrum test. The testing method is based on measuring the complex impedance over several decades of frequency. The results are compared to predictions of appropriate electrical equivalent circuits. Although several detection and analysis techniques for short circuits are known in the literature [5]-[7], the present approach is new.

The impedance spectrum test uses low-voltage. Therefore it cannot replace an insulation test with sufficient high AC voltage. One the other hand the impedance test is well suited to check for existing short circuits. Such short circuits may have been provoked by a preceding HV AC test. Depending on the resistance of the short circuit, the number of short-circuited turns and the frequency of the HV test, the short circuit may not be detected by the HV test itself. A variation of the frequency across the resonance frequency provides a higher sensitivity for short circuits. Since the impedance spectrum contains a lot of information, other applications to determine the electrical parameters of 
coils are possible e.g. check of inductance and capacity, resonance frequency and eddy current losses.

In the present work an automatic measurement of the impedance spectrum from $0.1 \mathrm{~Hz}-$ $100 \mathrm{kHz}$ has been performed by using a RLC-meter (HIOKI 3522-50) with RS-232 interface and software. For AC modeling a network of lumped elements is analyzed. When the resulting equivalent circuit is not too complicated the impedance can be represented by an analytic function. More complicated networks were analyzed with a network simulation code (SIMPLORER) [8]. Employing an analytic function for modeling has advantages over network analyzing codes because this function can be fitted to measured data. This allows one to determine unknown electrical parameters. For the same purpose the network simulation would need a time-consuming parameter variation. The results of the impedance spectrum test can serve to benchmark complex network analyses, in which many coils interact. In the case of W7-X e.g. the transient voltage over all 70 coils during a fast discharge of the current was modeled [8].

The methods presented in this paper can also be useful for similar components, i.e. coils or transformers in general. In particular for the fusion experiment ITER appropriate electrical testing methods have to be developed [9], [10].

The paper is organized as follows: In section 2 the electrical layout of the W7-X coils is presented. The modeling by network simulation and analytic functions is presented in section 3 and section 4, respectively. Section 5 focuses on the detection of short circuits. 


\section{Electrical layout of the W7-X field coils}

\section{a) Magnet system}

The stellarator W7-X consists of 5 symmetric modules. For the magnet system of W7-X 50 non-planar coils of five different coil types and 20 planar coils of two different coil types are needed [2]. Therefore 10 non-planar coils are built in a module, which are ordered folding-symmetrically around the half module centre. Figure 1 shows a half module of the W7-X magnet system with 5 non-planar and 2 planar coils. Plasma vessel, cryostat and support structure are not shown. Table 1 shows the main electrical parameters of the W7-X coils. The notation for the non-planar coils is AABxy and for the planar coils AACxy, whereas x denotes the module number and y the coil position within the module.

\section{b) Winding package}

The central component of the W7-X coils is an insulated and impregnated winding package, which is composed of the CIC (, cable in conduit ") superconductor of copperstabilized NbTi [11]. This package is embedded by means of a sand and epoxy resin mixture into a stainless steel casing.

The coil cross section of a non planar coil is shown in Figure 2. A non-planar coil consists of twelve layers formed by nine turns each. For the planar coils the winding pack is composed by 6 x 6 conductors. Two layers form a double layer (DL), which is wound from one superconductor length. All double layers are electrically connected in series by joints (see connection area in Figure 3). The coil terminals are layer 2 and 12 for the nonplanar coils and layer 3 and 6 for the planar coils respectively. For the He-cooling circuit 
the double layers are hydraulically connected in parallel. The cooling pipes are electrically separated from the winding by potential breakers. At each double layer quench detection cables are connected, which allow measuring the voltage drop over the individual double layers.

\section{c) Electrical insulation and high-voltage tests}

The insulation of the superconductor, the winding package, and the whole coil are designed to withstand the maximum expected voltages and mechanical stresses. The superconductors are insulated by fibre-glass reinforced epoxy-resin. The surface of the winding pack is covered by a $6 \mathrm{~mm}$ thick ground insulation. This is covered by an electrically conductive paint which is connected electrically with the coil casing (ground potential). The conductive paint serves for defining the electrical potential on the winding package surface. The quench detection cables are insulated with polyimide.

Ground isolation is tested with DC voltage on the superconductor against ground ( $\leq 13$ $\mathrm{kV})$. The insulation between adjacent layers is tested with AC or impulse voltage $(\leq 4$ $\mathrm{kV}_{\mathrm{pp}}$ ) applied on the winding terminals [4]. The frequency in HV-AC tests is $2 \mathrm{kHz}$ for the non-planar and $5 \mathrm{kHz}$ for the planar coils. The entire insulation is tested at ambient temperature in air and at cryogenic conditions in high vacuum. The ground insulation is even tested at different pressure levels at ambient temperature (Paschen test) to exclude arcing in residual gas under vacuum [12]. This means that the high voltage strength of the insulation is guaranteed in the entire pressure range between atmospheric pressure and high vacuum. 


\section{d) Coil casing}

The winding package of the coil is completely surrounded by a casing made of high grade steel. The casing is provided with cooling structures made from copper, in order to ensure a good cooling. They consist of copper stripes, which are tied up to circulating high grade steel pipes. This design is chosen in order to avoid high eddy currents during fast discharge of the coil current.

\section{AC modeling with network simulations}

For simplicity we will restrict our analysis to the planar coils in this section. The extension to non-planar coils is straightforward by just adding more elements corresponding to the higher number of conductors in the winding pack.

\section{a) Individual double layer}

The starting point of the simulation is inductance and capacity of a single turn. Taking for a planar coil an average conductor length of $390 \mathrm{~m}$ divided by 36 turns yields an average circumference of one turn $l=10.8 \mathrm{~m}$. The effective diameter $d$ of the CICC (cable-inconduit-conductor) cable is $18 \mathrm{~mm}$. The self-inductance of a circular loop is obtained by the formula [13]

$L[n H]=2 l[c m](\ln (l / d)-1.07)$.

This gives $L=11.5 \mu \mathrm{H}$ for one turn of the planar coils, which is used for the simulation. The capacity of adjacent turns within a layer is $6.4 \mathrm{nF}$, and of opposing turns of two layers $3.9 \mathrm{nF}$. These values are calculated from the formula for parallel plates $\left(\varepsilon_{\mathrm{r}}=5.8\right)$. 
With these values the network can be constructed like shown in Figure 4. The network calculation takes into account the coupling constants between all turn to turn combinations. These values range between 0.75 for adjacent turns and 0.48 for furthermost turns. The coupling constants are inferred from EFFI (electromagnetic fields, forces and inductances) calculations (see references in [3]). There is an identical spacing of the conductors inside the cross section of the winding pack for planar and non-planar coils. Therefore a subset of coupling constants for the non-planar coils is used for the planar coils. From the main resonance and the inductance at low frequency $(\sim 1 \mathrm{kHz})$ the capacity of the whole double layer is calculated, resulting in a value of $11 \mathrm{nF}$. This value is taken for the simulation of the planar winding pack shown in Figure 6 in which each double layer is treated as one $\mathrm{LC}$ element.

The network of Figure 4 was reduced in the number of elements (turns) to check the general relation between the number of turns and the total capacity. Figure 5 shows the result. The increase of the capacity is in good approximation linear with the number of turns.

\section{b) Bare planar winding pack}

The simulation network of a bare planar winding pack (without conductive paint or casing) is shown in Figure 6. The network calculation takes into account the coupling constants between the double layers, with the values of 0.89 for adjacent double layers and 0.77 for double layers 1 and 3. The simulation results are shown in Figure 7. This figure also compares the simulation with measurements and the analytic function for a bare winding pack presented in section 4.a). Measurements agree well with the simulation and the analytic function up to $\sim 50 \mathrm{kHz}$. For higher frequencies the simulation 
shows a second resonant peak which is not found in the measurements. Different trials with SIMPLORER show that these higher order resonances appear due to the capacities in between adjacent double layers (e.g. layer 4 in DL 2 to layer 5 in DL 3). When one removes these capacities, the higher order resonances vanish. However the resonance frequency increases about a factor of two, which would be in contradiction to the measurements. For this reason and because of the physical justification the capacities between adjacent double layers are kept in the simulation network. The question remains about the significance of the higher order resonances.

\section{AC modeling with analytic functions}

Figure 8 shows the equivalent circuits used for modeling the impedance spectra of coils in different states. The main electrical parameters are given in Table 1. Presenting the various equivalent circuits it will also be stated by which method a parameter was determined.

\section{a) Bare winding pack}

Figure 8a shows the equivalent circuit for a bare winding pack (without conductive paint or casing). The winding pack itself behaves as a parallel resonance circuit with the impedance:

$$
Z=\frac{1}{\frac{1}{j \omega L_{0}+R_{0}}+j \omega C_{0}} .
$$


The inductance $L_{0}$ of the winding with resistance $R_{0}$ acts in parallel to a capacity $C_{0}$. The inductance has been calculated by the EFFI code (see references in [3]). The winding resistance is only of importance for very low frequencies $\mathrm{f}<10 \mathrm{~Hz}$ and room temperature, otherwise it can be neglected because of $\omega L_{0}>>R_{0}$. The capacity is distributed over the whole winding. It can be calculated taking into account the network of all capacities between adjacent turns, as described in section 3 , or inferred from the resonance frequency of the winding pack.

\section{b) Winding pack with conductive paint}

Applying the conductive paint on the outer surface of the winding pack has the effect of an additional RC bypass (Figure $8 \mathrm{~b}$ ). This network has the impedance:

$$
Z=\frac{1}{\frac{1}{j \omega L_{0}+R_{0}}+j \omega C_{0}+\frac{\omega C_{1}}{\omega R_{1} C_{1}-j}} .
$$

The value $C_{1}$ depends on the area of the radial inner and outer side (first and last layer) of the winding pack surface and the ground insulation parameters. $R_{1}$ is the resistance of the conducting paint for a current flowing from inner to outer side. The effect of the conductive paint is that the sharp resonance is broadened and shifted to a lower frequency (see also Figure 9). The value of $R_{1}$ is obtained by fitting Equation (4b) to measured spectra. Alternatively, the resistance $R_{1}(l / u)^{2}$ across the large winding pack diameter has been measured on the surface of the conducting paint. The dimensions $l$ and $u$ denote large and small circumference of the winding pack (parallel and rectangular to the conductor direction). There is a huge scatter in $R_{1}$ for different winding packs, which can be explained by a high fabrication variability of the painting. 


\section{c) Winding pack with short circuit}

A short circuit in the winding can be represented by the equivalent circuit of an autotransformer (Figure 8c). This network has the impedance:

$$
Z=j \omega\left(L_{1}+M_{12}\right)+\frac{j \omega\left(M_{12}+L_{2}\right)\left(1-\frac{j \omega M_{12}}{R_{2}}\right)}{1+\frac{j \omega L_{2}}{R_{2}}} .
$$

The winding is separated into a short-circuited and non-short circuited part $\left(L_{1}\right.$ and $\left.L_{2}\right)$ with mutual inductance $M_{12}$. Self and mutual inductances can all be calculated with the EFFI code if the location of the short circuit and the number of turns involved are known. $R_{2}$ is the short circuit resistance. Under some circumstances this resistance can be measured independently by Ohmic measurements (e.g. short circuit with low resistance of a full double layer). Otherwise it has to be determined by fitting Equation (4c) to the measured impedance spectrum. In this equivalent circuit the winding resistance, the capacity and the conductive paint were neglected. They can easily be added into Figure $8 \mathrm{c}$ by adding the respective elements and modifying the equation accordingly. This treatment holds also for the following equations, which consider only the basic underlying equivalent circuits.

\section{d) Complete coil (in casing)}

The coil is completed by inserting the winding pack into the casing. The main effect of the casing is that it acts as an inductively coupled (single) transformer winding for the main primary winding (Figure 8d). The network has the impedance: 


$$
Z=R_{0}+j \omega L_{0}+\frac{\omega^{2} M_{03}^{2}}{R_{3}+j \omega L_{3}} .
$$

The self and mutual inductance of the casing, $L_{3}$ and $M_{03}$ respectively, were determined by the EFFI code and are given in Table 1. Mutual inductance and coupling factor $k$ are related by $M_{\mathrm{xy}}=k_{\mathrm{xy}}\left(L_{\mathrm{x}} L_{\mathrm{y}}\right)^{1 / 2} . R_{3}$ is the resistance of the casing for the eddy current flowing around the casing circumference. The casing leads to a reduction in $|Z|$ of almost one order of magnitude during the AC-HV tests. In addition to this, a much higher real power is needed than for tests on bare winding packs. This has to be considered in the design of the HV test equipment.

For later coil operation the low frequency part of Equation (4d) is important. The time scale of a fast current discharge is in the range of some seconds, corresponding to frequencies less than $1 \mathrm{~Hz}$. Even at this frequency, the eddy currents in the casing cannot be neglected. The casing resistance was treated as a free parameter and fitted to the measured spectra. The fitted values agree reasonably well with calculations (for nonplanar coils in simplified geometry). The specific resistance of the stainless steel at $4 \mathrm{~K}$ is $5 \cdot 10^{-7} \Omega \mathrm{m}$. The network of Figure $8 \mathrm{~d}$ is combined with network Figure $8 \mathrm{~b}$ to describe also the high frequency behavior (parallel connection of $C_{0}$ and $C_{1}, R_{1}$ ). Results are shown in Figure 12. Although the general agreement of measurement and calculation is good some deviations appear at frequencies above $\sim 100 \mathrm{~Hz}$ where the measured modulus and phase angle are smaller than calculated. This indicates that Equation (4d) does not include all eddy current losses. A space resolved model of the current distribution with a finite element code is proposed for further investigations. 


\section{e) Coil with short circuit}

A short circuit in a complete coil is inductively coupled to both the primary winding and the casing. Therefore three mutual inductances have to be considered in the equivalent circuit (Figure 8e). This network has the impedance:

$Z=j \omega\left(L_{1}+M_{12}\right)+\frac{\omega^{2}\left(M_{13}+M_{23}\right) M_{13}}{R_{3}+j \omega L_{3}}+j \omega \frac{\left(R_{2}-j \omega M_{12}-\frac{\omega^{2} M_{13} M_{23}}{R_{3}+j \omega L_{3}}\right)\left(M_{12}+L_{2}-\frac{j \omega\left(M_{13}+M_{23}\right) M_{23}}{R_{3}+j \omega L_{3}}\right)}{R_{2}+j \omega L_{2}+\frac{\omega^{2} M_{23}^{2}}{R_{3}+j \omega L_{3}}}$.

For the high frequency behavior see previous paragraph.

\section{Applications for short circuit detection}

In the course of the magnet production and testing the impedance spectrum test was established in 2005 to exclude or analyze short circuits within the winding packs [3]. Short circuits of a full double layer in three non-planar coils of two different types were observed within production before 2005. These short circuits were caused by an insufficient insulation in the crossing point of two conductors, combined with inappropriate pressing during vacuum impregnation. These short circuits could be repaired by excavating the respective area. One coil had been already inserted into the casing because the short circuit had not been found earlier due to insufficient testing. A risk for already existing coils of the same type remained that they could develop a short circuit during high-voltage or current tests. This was in fact the case for another coil in the year 2006, which led to the decision to excavate and insulate the critical areas in seven other coils with an inherent risk to develop such short circuits. 
Another type of short circuit was observed in only one non-planar winding pack

(AAB49). The short circuit was discovered by HV impulse testing when the winding pack had been vacuum-impregnated. This short circuit of probably a single turn was located deep inside the winding pack. It was possibly caused by a metallic chip. This winding pack could not be repaired and had to be replaced by a new one. We will analyze both short circuits in this section.

The impedance test is most sensitive to short circuits when the frequency is close to the main resonance frequency. When the winding pack has already been inserted into the casing, a short circuit is masked to a large extent by the eddy currents in the casing. This is especially the case when the short circuit comprises only one winding. Modeling is important to check the detection limits of the test. In the following, impedance spectra of the two types of short circuits found in the W7-X non-planar coils will be presented.

\section{a) Winding pack}

Figure 9 shows spectra measured on non-planar winding packs (AAB33 bare, AAB33 with conductive paint, AAB49 with short circuit), corresponding to equivalent circuits of Figure 8a-c. Points denote measured values; lines denote curves according to the Equations (4.a)- (4.c) with the parameters given in Table 1 and Table 2. The spectrum of a bare winding pack has a very sharp resonance. Applying the conductive paint shifts this resonance to a lower frequency because of the additional capacity. The damping of the resonance is caused by the finite resistance of the conductive paint. In contrast to this, the short circuit shifts the resonance to a higher frequency. Due to the real power within the short circuit, the complex $Z$ contains a large real part and therefore the phase angle tends to smaller values than without a short circuit. The short circuit resistance $R_{2}$ and the 
coupling constant $k_{12}$ were obtained from fitting the measured data (EFFI calculations however result in a somewhat smaller coupling constant $k_{12}$, depending on the short circuit location). The relatively high resistance of the short circuit indicates that the contact area of the short circuit is in the sub-mm range. Therefore the origin could be a small metallic chip. The conclusion that the short circuit comprises only one turn is based on the following fact: It is impossible to match model and observed spectra with assuming a short circuit of more than one turn, when a parameter variation of $R_{2}$ and $k_{12}$ is performed. This means that two adjacent turns in the same layer (or at the layer transition) have a short circuit.

Figure 10 shows the impedance at a fixed frequency measured over individual winding pack double layers. The impedances of the intact winding pack double layers increase from inner to outer layer, as it is expected because of the larger inductance at larger radius. In contrast to this, the impedance of the winding pack with a short circuit has a minimum in double layer 4 . This indicates the location of the short circuit. The coupling of the short circuit is strongest in the adjacent double layers 3 and 5. Since the impedance for DL 5 is lower than that of DL 3 it can be concluded that the short circuit is in the outer layer of DL 4 which is adjacent to DL 5. The short circuit could not be repaired since it was not possible to excavate DL 4 in sufficient depth without destroying the winding pack (see Figure 11). Hence this winding pack (AAB49) had to be replaced.

Obviously this type of short circuit with a single turn and a relative high contact resistance can only be detected if the test frequency is large enough (i.e. $>1 \mathrm{kHz}$ ). In general, impedance measurements at fixed frequency (e.g. standard $50 \mathrm{~Hz}$ ) seem to be not appropriate for short circuit detection. 


\section{b) Complete coil}

One non-planar coil (AAB25) developed a short circuit during cold tests. The cold test procedure [4] comprises tests nominal current and elevated temperature $(5.7 \mathrm{~K}$ for the non-planar coils) to check the safety margin of the superconductor, flow tests, leak tests, and high voltage tests presented in section 2.c). After 10 load cycles at nominal current a HV-AC test with $3.2 \mathrm{kV}_{\mathrm{pp}}$ has been applied. An overload of the AC power supply occurred and the coil failed that test. The impedance spectrum was recorded and showed strong deviations from the initial spectrum. All measurements were performed at cryogenic temperature $(\sim 10 \mathrm{~K})$, slightly above the critical temperature of superconductivity. Figure 12 shows the comparison of measurements and calculation for the coil AAB25 before and after the short circuit of a full double layer. The short circuit manifests itself as a strong distortion of the spectrum in the whole frequency range. In addition to the impedance tests, resistance measurements on the individual double layers were performed at the beginning and at the end of the cold tests. It turned out that the resistance of DL 2 had dropped to only $0.16 \mathrm{~m} \Omega$ instead of an expected value of 0.23 $\mathrm{m} \Omega$. From these measurements it could be concluded that a short circuit in DL 2 had occurred, with a short circuit resistance $R_{2}$ of $0.5 \mathrm{~m} \Omega$ (in parallel to DL 2). These values were used as input for the calculated spectra. As analytic functions, Eq. (4.c) and (4.e) were applied and combined with Eq. (4.b) (parallel network). The parameters given in Table 1 and Table 2 were employed. Measurements and calculations agree fairly well, especially in the low frequency range below $10 \mathrm{~Hz}$. 
After warm-up the coil was sent to the manufacturer where the short circuit could be found and removed. It was in the crossing area of layer 3 and 4 where the insulation had not been sufficient (see also Figure 3).

Figure 13 shows measured impedance spectra of ten different coils at cryogenic temperature. All measurements were performed by adding a parallel capacitance of $47 \mathrm{nF}$ in order to limit the scatter due to variations in coil capacities. The shape of the envelope curve agrees well with the calculation shown in Figure 12. If one compares absolute values one notes however that measured phase angle and resonance frequency are somewhat smaller than calculated. For a sensitivity study of short circuit detection we only use the calculations to predict relative changes in the spectrum induced by the short circuit. In the measurements, especially for frequencies above $200 \mathrm{~Hz}$, only a small scatter is observed. Therefore a high sensitivity for short circuits is expected.

\section{c) Detection limit}

The question arises what short circuits could be detected with the impedance spectrum test. Systematic calculations and measurements (see also [3]) were performed to investigate the detection limits. Obviously, in contrast to resistance measurements, the impedance spectrum is much more sensitive. The sensitivity is generally large when

(i) the short circuit involves a lot of turns

(ii) the short circuit is of low resistance

(iii) the bare winding pack is measured

The first two points are based on the Ohmic power within the short circuit $U^{2} / R$ which increases quadratically with the number of turns and decreases with short circuit 
resistance. The third point is based on the fact that any additional losses mask the effect of the short circuit. The casing can be regarded as an (external) short circuit of very low resistance. Even a surrounding closed loop by a metallic support frame can present a disturbance of the spectrum.

Single turn short circuits are the most difficult ones to detect. Their influence on the spectrum depends on the location within the winding pack: turns in the center have a higher coupling to the rest of the winding pack than turns at the edge. The latter one presents the worst-case; for this case systematic studies of the detection limit were performed and presented in Table 3. A more likely case is however the short circuit of a full double layer in the coil connection area as presented in the previous subsection. For this case the detection limit was determined as well and summarized in Table 3 . The value given in Table 3 for the resistance means that a short circuit having a smaller resistance than this value can be detected safely. This resistance limit depends on the coil type (planar, non-planar), location of the short circuit and other properties of the coil which may have large production variability (e.g. the resistance of the conductive paint). Therefore only an order of magnitude is given. It is important to note that short circuits in single turns can only be detected safely on the bare winding pack. Therefore impedance tests at the manufacturer are especially important. On the other hand it is very unlikely that a single turn short circuit will be caused by high voltage occurring in test or operation of the coil because the inter-turn voltage is always low. More critical in this respect are short circuits of double layers where the voltage drop is much larger.

Let us now consider the heating power dissipating in a short circuit. In a safety discharge of W7-X the voltage across a non-planar coil is $\mathrm{U}_{\max } \sim 400 \mathrm{~V}$, corresponding to a voltage 
across one turn of $\sim 4 \mathrm{~V}$ and $\sim 70 \mathrm{~V}$ across a double layer. Assuming for the heating power $\mathrm{U}^{2} / \mathrm{R}_{2}$ and the parameters in Table 2, a maximum power of $\sim 160 \mathrm{~W}$ for the single turn but $\sim 10 \mathrm{MW}$ for the double layer short circuit, respectively, is deposited into the conductor. A destruction of the coil can happen either by the rapid heating itself, or by an unbalanced current which leads to forces exceeding the design limits. It is self-evident that a short circuit of the latter type (low-Ohmic, in double layer) will probably destroy the coil while the afore-mentioned type (in single turn) might be tolerable. Short circuits with a relatively high resistance would probably not present serious problems during current ramp-up or fast discharge. Therefore the present detection limit seems to be acceptable. Short circuits with a lower resistance, more turns, or when they occur on winding packs not embedded, produce larger deviations in the impedance spectrum and can be clearly identified.

\section{Conclusions}

Substantial progress has been achieved over the last years in the application and interpretation of electrical tests with $\mathrm{AC}$ voltage on the W7-X coils. Key elements are that appropriate tests are carried out after each relevant step in production or acceptance.

The impedance spectrum test can exclude short circuits in the W7-X magnets. Its sensitivity is better than that of ordinary AC tests and DC resistance measurements. Since the sensitivity decreases when the coil casing is present the test should be performed also on the bare winding pack. The impedance spectrum test is a non-destructive test and is therefore well suited for frequent checks. Nevertheless, an AC test with high voltage 
remains essential at different stages of the test program in order to apply a dielectric stress to the inter-turn insulation. Since the short circuit resistance is generally smaller at a lower temperature, the impedance test is more sensitive at cryogenic temperature. The test should therefore be obligatory within the cold test procedure [4], after all other tests which apply mechanical or dielectric stress on the insulation (high current and high voltage) have been completed. The combination of high-voltage AC and low voltage impedance spectrum tests are therefore ideal means to rule out short circuits in the W7-X coils.

The impedance spectrum test provides also a lot of information about electrical parameters when being interpreted by appropriate electrical schemes. This information is valuable when predicting the electrical behavior in transient or off-normal events. The experience gained with the $\mathrm{W} 7-\mathrm{X}$ magnet testing provides a good basis to define test requirements and procedures for the magnets of ITER. The present analysis might even be valuable for components with similar electrical features (normal-conducting coils, transformers, etc). 


\section{References}

[1] M. Wanner, V. Erckmann and J.H, Feist, Nuclear Fusion 43 (2003), 416

[2] C. Sborchia, J. Baldzuhn, J.H. Feist, K. Riße, T. Rummel, H. Viebke and M. Wanner, IEEE Trans. Appl. Supercond. 16 (2006), 848.

[3] H. Ehmler, H. Fillunger, J. Baldzuhn, R.K. Maix, A. Jeckle, S. Parodi, T. Rummel, K. Riße and H. Scheller, IEEE Trans. Appl. Supercond. 16 (2006), 767.

[4] H. Ehmler, J. Baldzuhn, C. Sborchia, L. Genini, T. Schild and K. Heyn,"Review of the Acceptance Tests of the W7-X Superconducting Magnets”, Fus. Eng. \& Design, article in press: http://dx.doi.org/10.1016/j.fusengdes.2007.01.018.

[5] Y. Iwasa, M. J. Leopold, R. J. Weggel, J. R. Hale and J.E.C. Williams, IEEE Trans. on Magnetics, 19 (1983), 704

[6] P. L. Walstrom, IEEE Trans. on Magnetics, 19 (1983), 1094

[7] E. Acerbi, G. Baccglioni, G. C. Cartegni, M. Sorbi and G. Volpini, IEEE Trans. Appl. Supercond. 14 (2004), 1414.

[8] M. Köppen, H. Kißlinger, "Simulation of Voltage and Current Development in the W7-X Coil System Taking into Account Fault Conditions”, Fus. Eng. \& Design, article in press: http://dx.doi.org/10.1016/j.fusengdes.2007.01.010

[9] M. Matsukawa, Y. M. Miura and K. Shimada, IEEE Trans. Appl. Supercond. 14 (2004), 1414. 
[10] S. Fink, T. Boncelli, W. H. Fietz, A. Miri, X. Quan, A. Ulbricht., Fusion Engineering \& Design 75-79 (2005), 135

[11] R.K. Maix, V. Bagnato, M. Fricke, K. Heyn, T. Kluck, F. Lange, K. Riße, C.

Sborchia and N. Valle, Journal of Physics: Conference Series 43 (2006), 753, 7th European Conference on Applied Superconductivity

[12] H. Scheller, H.P. Langenberg, M. Kühnberg, J. Baldzuhn, B. Petersen-Zarling and D. Gustke et al., IEEE Trans. Appl. Supercond. 16 (2006), 759.

[13] H. Meinke and F. W. Gundlach, Book:,,Taschenbuch der Hochfrequenztechnik“, Springer (1968) 


\section{Tables}

Table 1: Main electrical parameters of the W7-X magnetic field coils.

\begin{tabular}{|c|c|c|c|}
\hline & Symbol & Non-planar coil & Planar coil \\
\hline Number of turns & & 108 & 36 \\
\hline Number of double layers & & 6 & 3 \\
\hline Average diameter & $l /(2 \pi)$ & $3.0 \mathrm{~m}$ & $3.3 \mathrm{~m}$ \\
\hline Rated current & & $17.6 \mathrm{kA}$ & $16 \mathrm{kA}$ \\
\hline Insulation DC voltage (winding-ground) & & $6 \mathrm{kV}$ & $4 \mathrm{kV}$ \\
\hline Maximum terminal voltage (AC) & & $1 \mathrm{kV}$ & $1 \mathrm{kV}$ \\
\hline Winding resistance at $4 \mathrm{~K}$ & $R_{0}$ & $<6 \mathrm{n} \Omega$ & $<2.5 \mathrm{n} \Omega$ \\
\hline Winding resistance at $300 \mathrm{~K}$ & $R_{0}$ & $0.13 \Omega$ & $0.05 \Omega$ \\
\hline Winding self-inductance & $L_{0}$ & $0.05 \mathrm{H}$ & $0.009 \mathrm{H}$ \\
\hline Winding distributed capacity & $C_{0}$ & $5 \mathrm{nF}$ & $12 \mathrm{nF}$ \\
\hline Conducting paint resistance (section 4.b) & $R_{1}$ & $(0.5-2.5) \mathrm{k} \Omega$ & $(0.4-8) \mathrm{k} \Omega$ \\
\hline Conducting paint capacity (section $4 . b$ ) & $C_{1}$ & $6.5 \mathrm{nF}$ & $1 \mathrm{nF}$ \\
\hline Casing resistance at $4 \mathrm{~K}$ & $R_{3}$ & $0.1 \mathrm{~m} \Omega$ & $0.3 \mathrm{~m} \Omega$ \\
\hline Casing self-inductance & $L_{3}$ & $3.3 \mu \mathrm{H}$ & $5.5 \mu \mathrm{H}$ \\
\hline Winding-casing coupling factor & $k_{03}$ & 0.88 & 0.90 \\
\hline Winding casing mutual inductance & $M_{03}$ & $0.36 \mathrm{mH}$ & $0.2 \mathrm{mH}$ \\
\hline
\end{tabular}


Table 2: Parameters of short circuits detected in W7-X non-planar coils. Coil AAB25 developed a short circuit of a double layer after the current test (see also Figure 12). Winding pack AAB49 had a short circuit of one turn, which was detected on the bare winding pack (see also Figure 9 and Figure 10).

\begin{tabular}{|l|c|c|c|}
\hline & Symbol & AAB25 & AAB49 \\
\hline \hline Number of turns short circuited & & 18 & 1 \\
\hline Inductance of primary winding & $L_{1}$ & $36 \mathrm{mH}$ & $50 \mathrm{mH}$ \\
\hline Inductance of short circuited winding & $L_{2}$ & $1.7 \mathrm{mH}$ & $8 \mu \mathrm{H}$ \\
\hline Coupling factor primary- short circuit & $k_{12}$ & 0.85 & 0.92 \\
\hline Coupling factor primary- casing & $k_{13}$ & 0.89 & - \\
\hline Coupling factor short circuit- casing & $k_{23}$ & 0.79 & - \\
\hline Short circuit resistance & $R_{2}$ & $0.5 \mathrm{~m} \Omega$ & $0.1 \Omega$ \\
\hline
\end{tabular}


Table 3: Short circuit detection limit for winding packs in different production stages, summarizing the experience from model calculations and experiments. The maximum resistance of the short circuit is given as an order of magnitude at which the short circuit can safely be detected by the impedance spectrum test.

\begin{tabular}{|l|l|l|}
\hline \multirow{2}{*}{} & \multicolumn{2}{|c|}{ Short circuit detectable } \\
& \multicolumn{2}{|c|}{ (Resistance limit) } \\
\hline & In double layer & In single turn \\
\hline Bare winding pack & Yes $(<1000 \Omega)$ & Yes $(<1 \Omega)$ \\
\hline $\begin{array}{l}\text { Winding pack with } \\
\text { conductive paint }\end{array}$ & Yes $(<100 \Omega)$ & Very limited $(<0.01 \Omega)$ \\
\hline Winding pack with casing & Yes $(<10 \Omega)$ & No $^{1}$ \\
\hline
\end{tabular}

${ }^{1}$ Depending on short circuit location and resistance. 


\section{Figures}

Figure 1: Half module of the W7-X magnet system with 5 non-planar (NPC) and 2 planar coils (PC). Plasma vessel, cryostat and support structure are not shown.

Figure 2: Non planar coil cross section ( $12 \times 9$ conductor turns). The planar coils have a cross section of $6 \times 6$ conductors.

Figure 3: Connection area of non-planar winding pack. Layer 2 and 12 are the coil terminals.

Figure 4: Network model of an individual double layer of a planar coil ( $2 \times 6$ turns).

Figure 5: Total capacity of double layer (network shown in Figure 4) when the number of turns is varied.

Figure 6: Network model of planar winding pack (3 double layers).

Figure 7: Simulation, analytic function and measurements for a planar coil without conductive paint (AAC34).

Figure 8: Basic equivalent circuits. Winding packs: (a) bare (without conductive paint), (b) with conductive paint, (c) with internal short circuit (only low frequency). Coil in casing (only low frequency): (d) without internal short circuit, (e) with internal short circuit.

Figure 9: Impedance spectra of winding packs at ambient temperature corresponding to Figure 8 (a)-(c). Points denote measured values; lines denote curves according to the Equations $3 \mathrm{a}-3 \mathrm{c}$ with the parameters given in Table 1 and Table 2. 
Figure 10: Impedance (modulus) at fixed frequency measured over individual winding pack double layers. The minimum indicates the location of the short circuit.

Figure 11: Winding pack AAB49 with short circuit. The ground insulation has been removed and some conductors have been untied by cutting the insulation. This operation was not successful, the short circuit remained and the winding pack had to be abandoned.

Figure 12: Comparison of measurements and calculation for a coil in casing with and without short circuit of a full double layer. All measurements were performed at cryogenic temperature $(\sim 10 \mathrm{~K})$.

Figure 13: Impedance spectra of 10 different non-planar coils at cryogenic temperature. The detection sensitivity for short circuits is high where the deviations are small. 


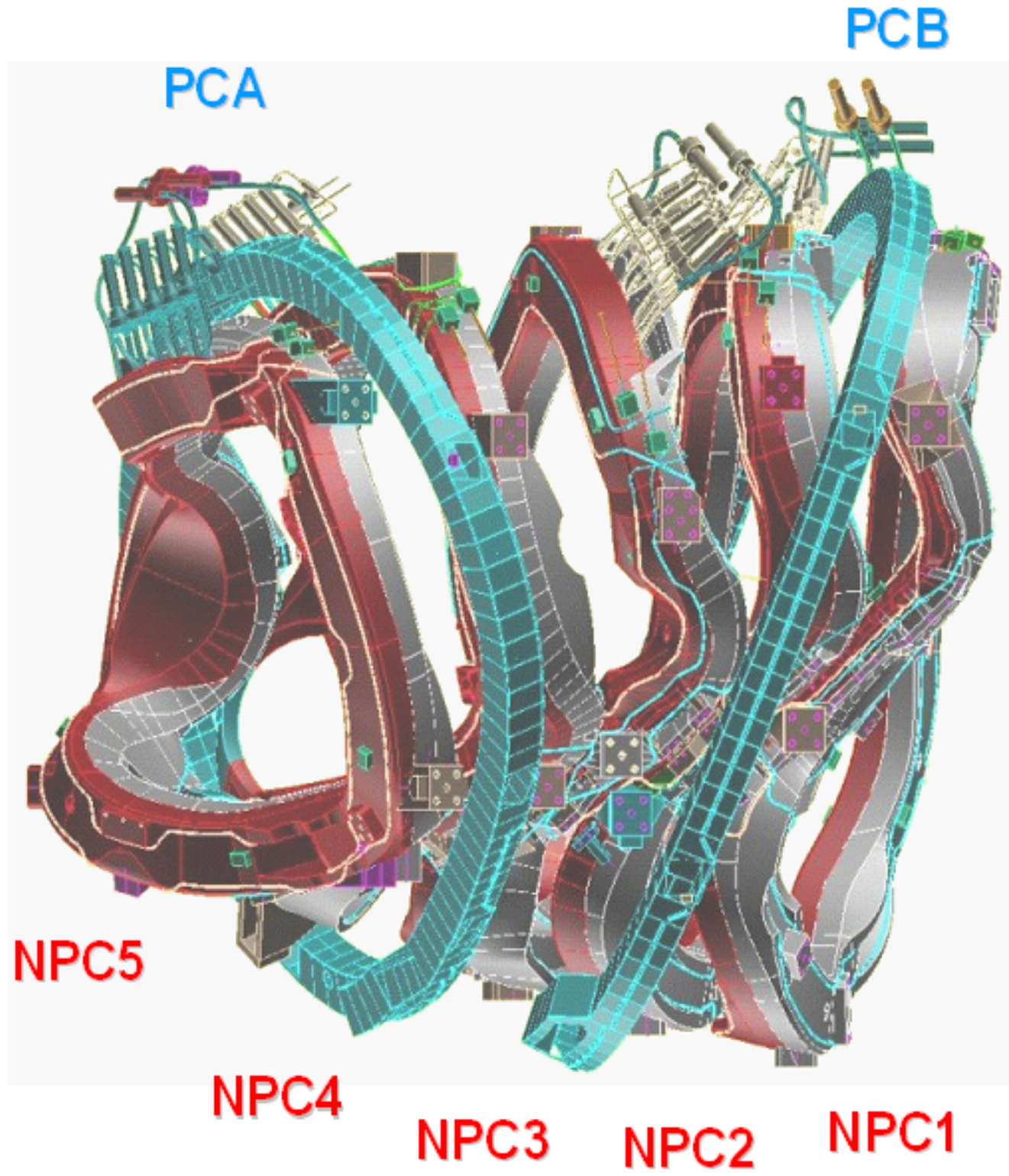




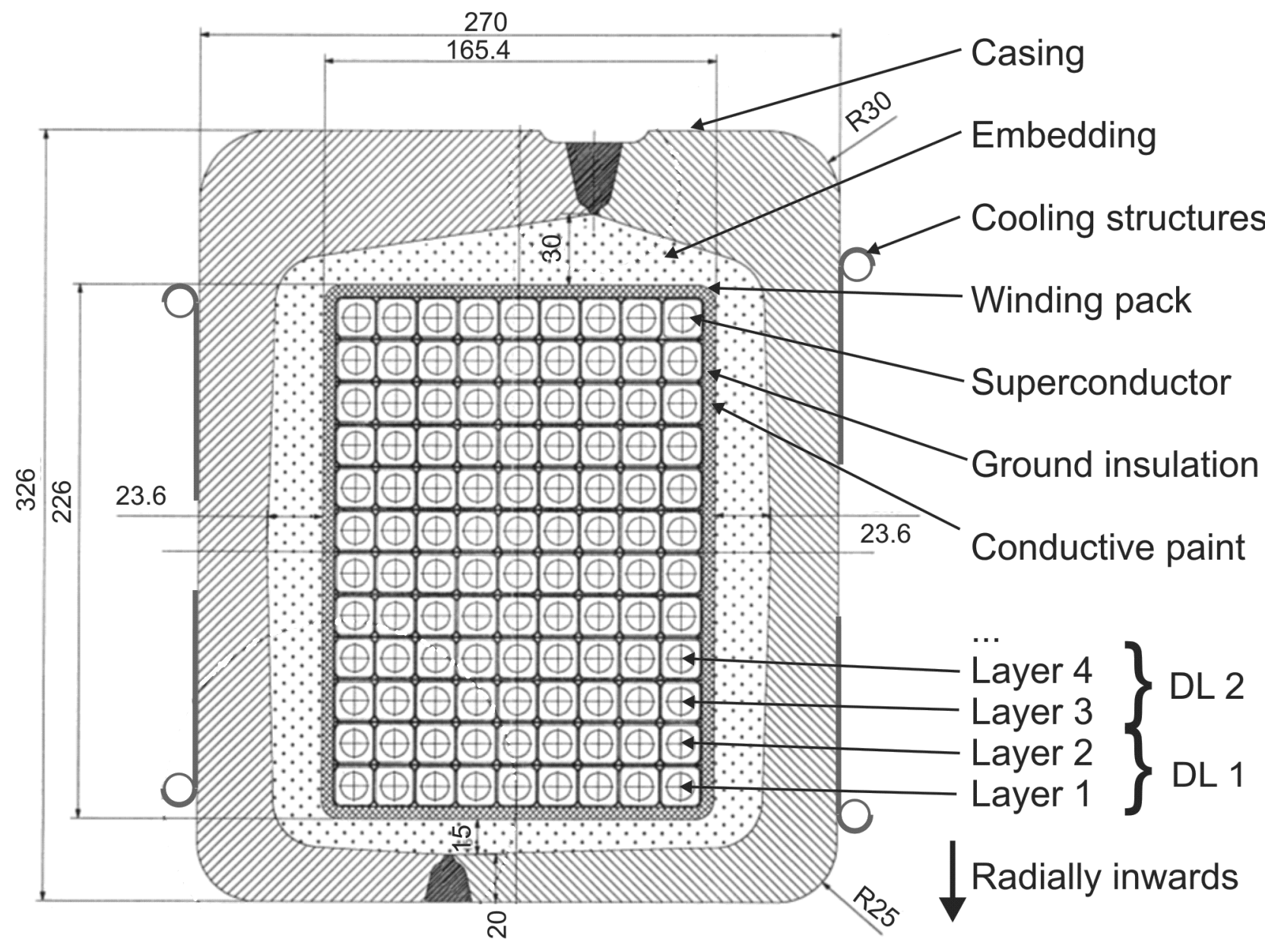




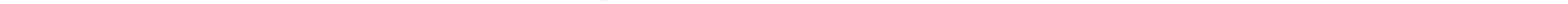




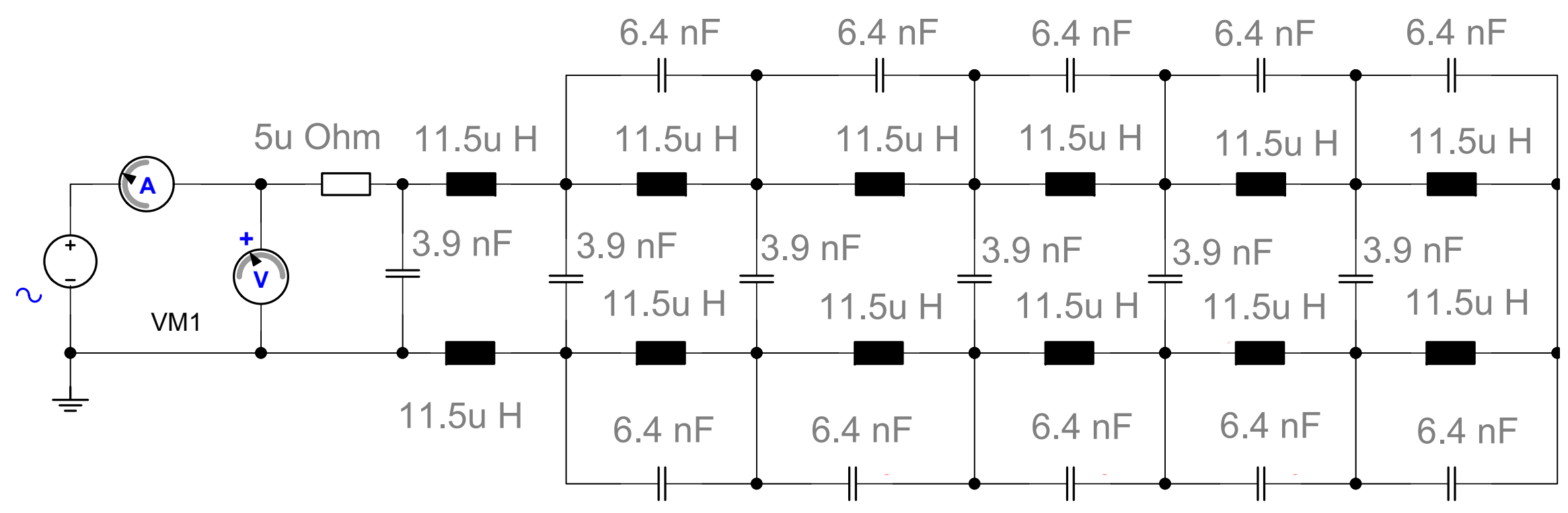




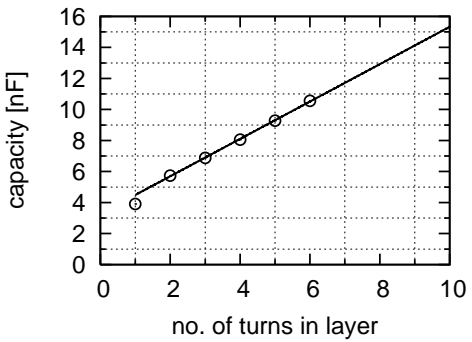




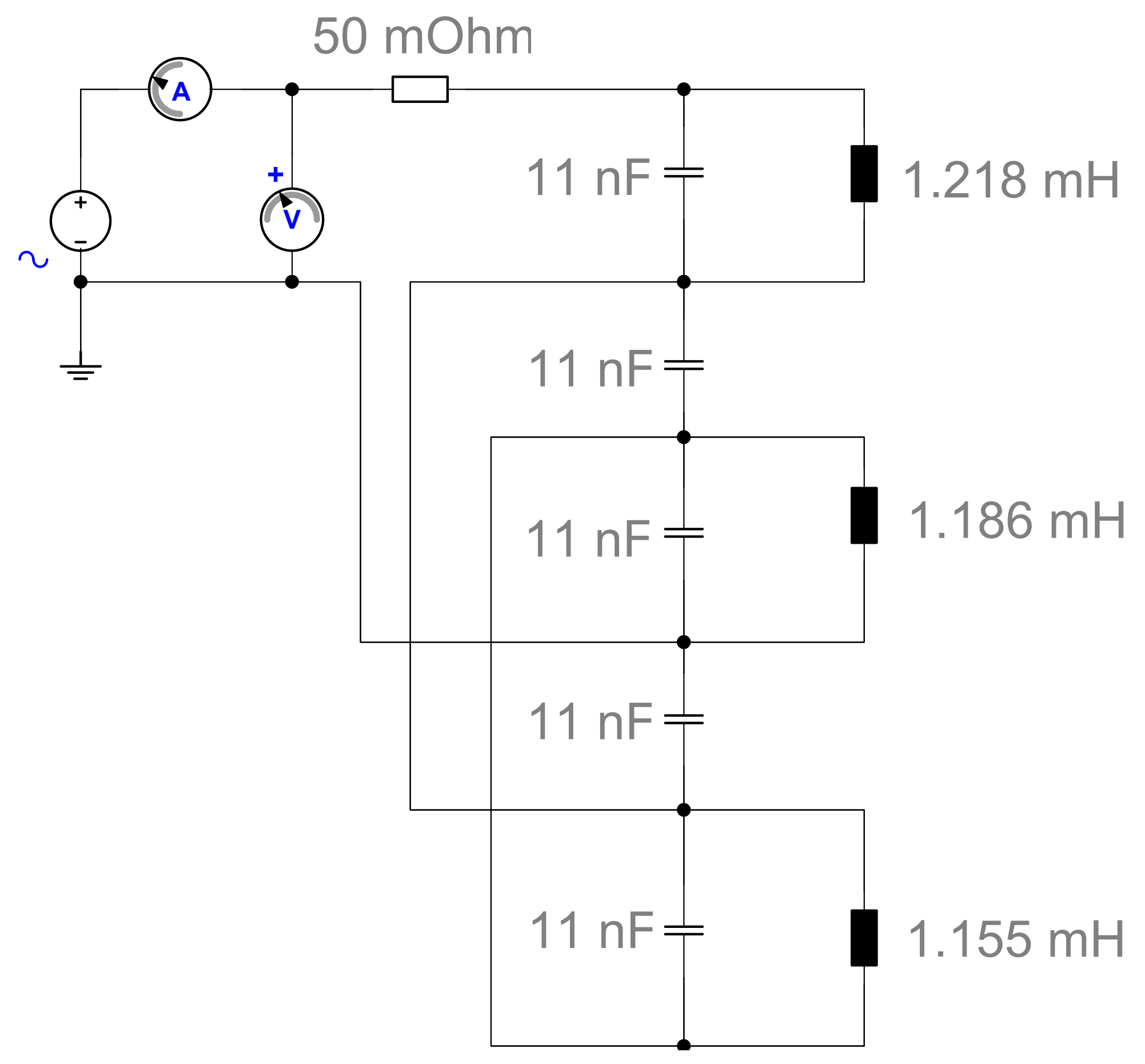



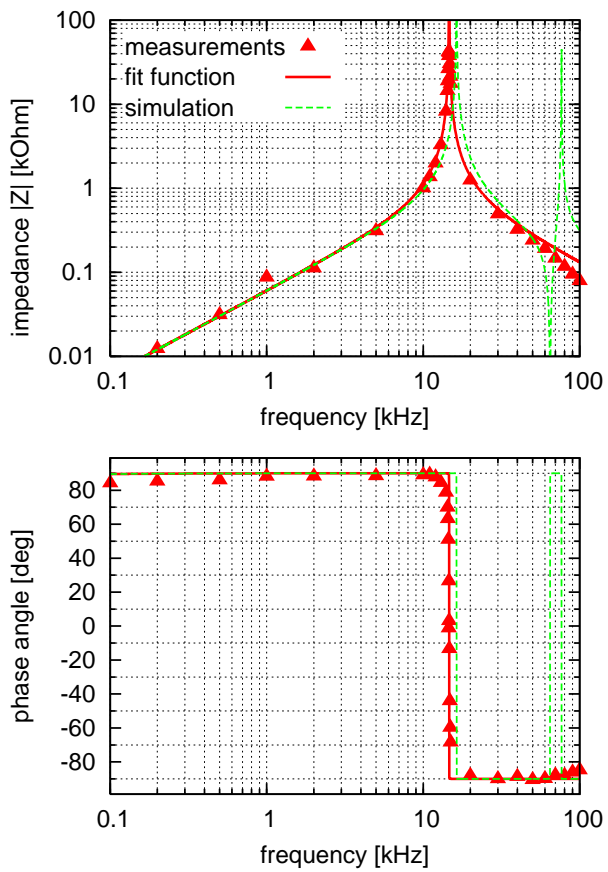

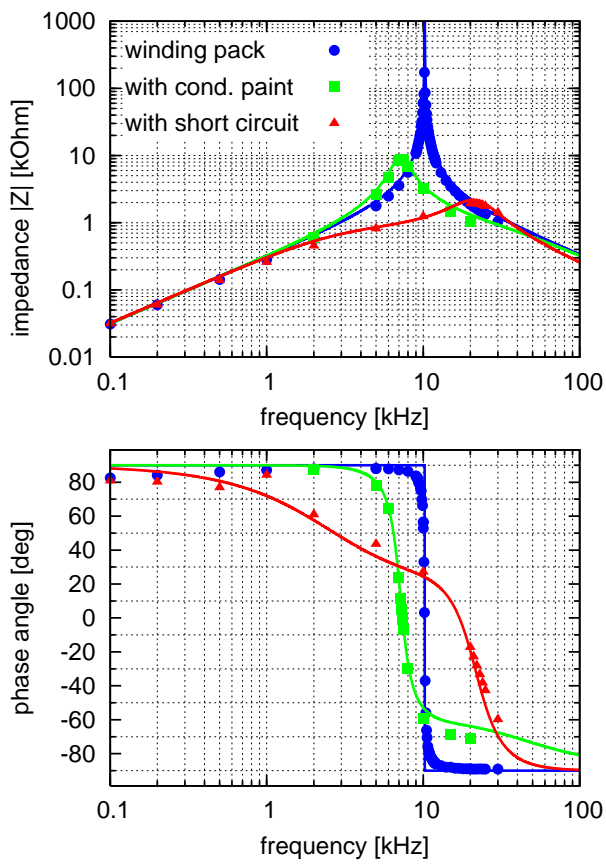


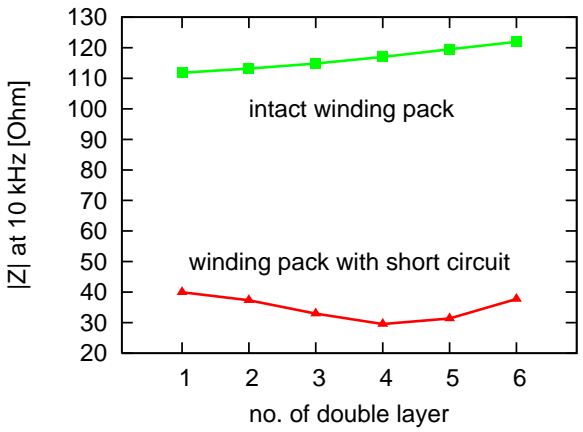




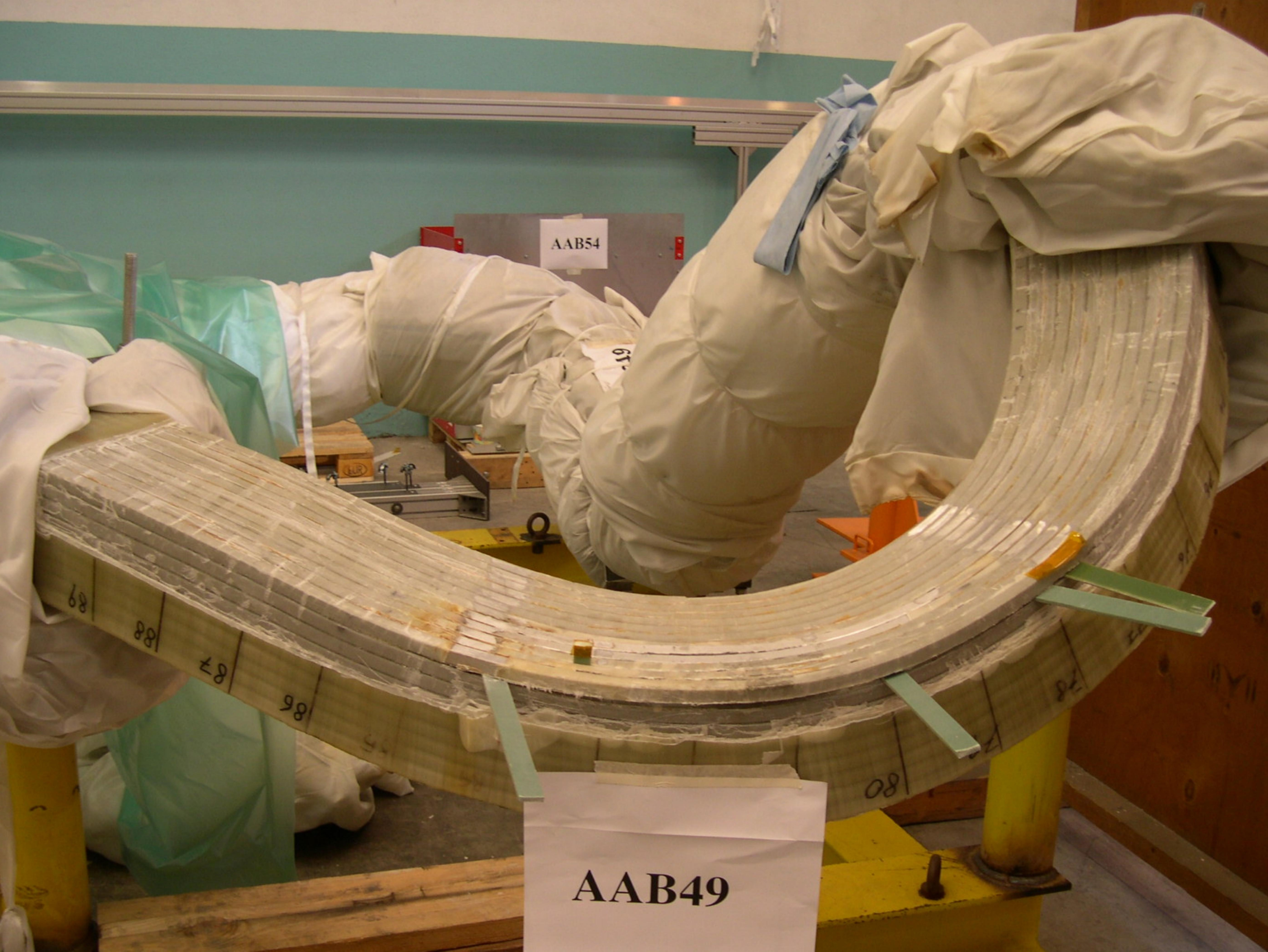



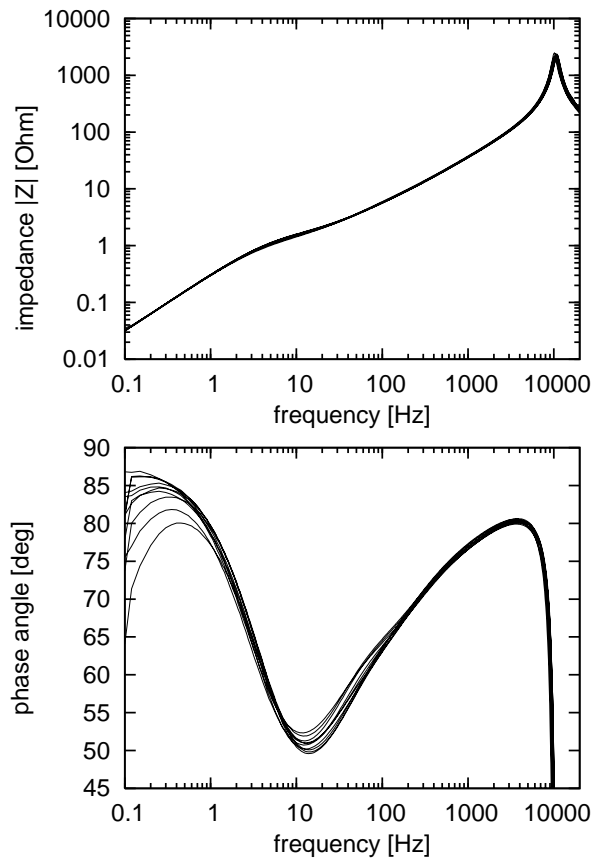